\title{
The Influence of Previous Antipsychotic Polypharmacy Versus Monotherapy on the Effectiveness of Antipsychotic after Switching to Paliperidone Extended- release
}

\author{
Hee-Won Lee', Kyoung-Sae Na², Seung-Ho Jung ${ }^{1}$, Min-Hee Kang', Jeong Seop Lee', Jae-Nam Bae', Hee-Yun \\ $\mathrm{Kim}^{1}$, Chul-Eung Kim \\ 'Department of Psychiatry, Inha University Hospital, Incheon, 'Department of Psychiatry, Soonchunhyang University Bucheon Hospital, \\ Bucheon, Korea
}

\begin{abstract}
Objective: Although antipsychotic polypharmacy is widely used in the pharmacotherapy of schizophrenia, its effectiveness is controversial. In particular, clinicians tend to avoid switching to monotherapy in patients who have been prescribed polypharmacy. In the present study, the authors investigate whether there is difference in time to discontinuation of antipsychotics between patients on previous monotherapy or polypharmacy.

Methods: Pooled analysis was conducted on two 24-week, multicenter, open-label, non-comparative studies that were originally designed to investigate the effectiveness of switching to paliperidone extended-release (ER) in patients with schizophrenia. Patients were divided into two groups according to previously prescribed antipsychotics, that is, to a polypharmacy group or a monotherapy group. The primary outcome measure was time to discontinuation of paliperidone ER. In addition, the authors sought to identify clinical variables that influence time to discontinuation.

Results: Before switching to paliperidone ER, 535 of 673 (79.5\%) patients were prescribed antipsychotic monotherapy, and the remaining 138 (20.5\%) patients were prescribed antipsychotic polypharmacy. No significant differences in time to discontinuation of paliperidone ER were observed between the polypharmacy and monotherapy groups. Personal and social performance scale score was the only factor found to influence time to discontinuation of paliperidone ER. No differences in psychopathology or adverse effects were found between the monotherapy and polypharmacy groups.

Conclusion: Our results suggest that number of antipsychotics prescribed before switching to monotherapy does not influence clinical prognosis in patients with schizophrenia.
\end{abstract}

KEY WORDS: Schizophrenia; Antipsychotic agents; Polypharmacy; 9-Hydroxyrisperidone, Treatment outcome.

\section{INTRODUCTION}

Schizophrenia is characterized by positive and negative symptoms, a decline in cognitive function, and mood symptoms. These symptoms wax and wane during clinical course and progressively affect social and occupational functions. ${ }^{1)}$ Pharmacotherapy is a major treatment strategy for schizophrenia, ${ }^{2)}$ and most guidelines recommend monotherapy. ${ }^{2-5)}$ Antipsychotic polypharmacy is recommended only after unsuccessful attempts with multiple

Received: July 15, 2013 / Revised: July 28, 2013

Accepted: August 2, 2013

Address for correspondence: Chul-Eung Kim, MD, PhD Department and Research Instutitute of Psychiatry, Inha University College of Medicine, 7-206, Sinheung-dong 3-ga, Jung-gu, Incheon 400-711, Korea

Tel: +82-32-890-3475, Fax: +82-32-890-3558

E-mail: kce320@inha.ac.kr monotherapies, including clozapine, which is known to have limited clinical efficacy. ${ }^{6}$ ) However, antipsychotic polypharmacy is known to increase side-effects and treatment cost, whereas it has an efficacy similar to that of monotherapy.

However, contrary to guidelines, antipsychotic polypharmacy is widely used in real clinical practice. ${ }^{6,7)}$ Clinicians tend to believe that polypharmacy is the better option, because it can reduce psychotic symptoms and total medication dose in those who do not respond to monotherapy. It is also assumed that change from polypharmacy to monotherapy exacerbates symptoms and leads to higher rates of medication discontinuation. ${ }^{8}$

Although few studies have conducted on the subject, previous studies have revealed that switching from polypharmacy to monotherapy does not aggravate clinical symptoms. ${ }^{9010)}$ In fact, Suzuki et al. ${ }^{9)}$ reported that $77.2 \%$

(c) This is an Open-Access article distributed under the terms of the Creative Commons Attribution Non-Commercial License (http://creativecommons.org/licenses/by-nc/3.0) which permits unrestricted non-commercial use, distribution, and reproduction in any medium, provided the original work is properly cited. 
of patients showed similar or better results after switching from polypharmacy to monotherapy, and Essock et al. ${ }^{10)}$ found that switching fastened discontinuation times of antipsychotics significantly and decreased body weights, but did not affect clinical symptoms. However, these two studies involved small sample sizes and for antipsychotic monotherpy, they chose to stop one of the current medications. Thus, the conclusion was made that existing polypharmacy might involve the prescription of unnecessary and redundant medicines.

In clinical practice, when there is a need to change antipsychotics, some clinicians tend to discontinue current antipsychotics, regardless of whether patients have taken monotherapy or polypharmacy, and try another new type of medication. ${ }^{11)}$ Hence, it is important to determine whether clinical efficacies are differentially affected after switching antipsychotics in patients who have previously taken antipsychotic monotherapy or polypharmacy.

In this study, we conducted pooled analysis to determine whether previous antipsychotic monotherapy or polypharmacy differentially influences time to discontinuation and clinical symptoms after switching to paliperidone extended-release (ER).

\section{METHODS}

\section{Subjects and Procedures}

We performed a pooled analysis of two 24 -week, multicenter, open-label, non-comparative studies in patients with schizophrenia. This study's protocol was reviewed and approved by the institutional review board of each participating hospital, and all patients gave written informed consent. Detailed descriptions of subject inclusion and exclusion criteria and the protocols used are described at ClinicalTrials.gov (NCT00761605, NCT00784238). Briefly, the original studies were conducted in men and women aged between 18 and 65 diagnosed with schizophrenia in accordance with criteria in the Diagnostic and Statistical Manual of Mental Disorders fourth edition (DSM-IV). ${ }^{1)}$ Patients that required switching to other antipsychotic medication due to lack of efficacy, a lack of drug tolerance, or a lack of compliance were selected among patients who had been treated with therapeutic medication for schizophrenia over 2 weeks prior to screening.

The exclusion criteria applied were as follows: a previous history of neuroleptic malignant syndrome, a history of allergic or hypersensitive reaction to risperidone or paliperidone, the receipt of clozapine within a month of screening. Patients were required to switch from existing antipsychotics to paliperidone ER. Although the recommended dosage was $6 \mathrm{mg}$ per day, dosages within the range 3 to $12 \mathrm{mg}$ were allowed based on clinician's judgment. No other antipsychotic medication was allowed during these studies.

For the pooled analysis, we divided the 673 study subjects into two groups according to the number of previous antipsychotics, that is, to a (1) monotherapy group $(\mathrm{n}=$ 535), members of which had previously taken antipsychotic monotherapy, and to a (2) polypharmacy group $(n=138)$, members of which had previously taken antipsychotic polypharmacy (Fig. 1). The primary outcome measure was time to discontinuation. In addition, the severities of psychopathology, psychosocial function, and extrapyramidal symptoms (EPS) in the two groups were compared during 24 weeks.

The Krawiecka scale (KS) was used for measuring psychiatric symptoms. ${ }^{12)}$ This scale provides a straightforward and reliable means of assessing the symptoms of patients with schizophrenia. It makes assessments by classifying symptoms, such as, positive symptoms, negative symptoms, depression, and anxiety into eight items. Of these eight items, four (delusion, hallucination, depression, and anxiety) are assessed by patients themselves, whereas the other four (incoherence, poverty of speech, flattened affect, and psychomotor retardation) are assessed by a trained rater.

We used the personal and social performance scale

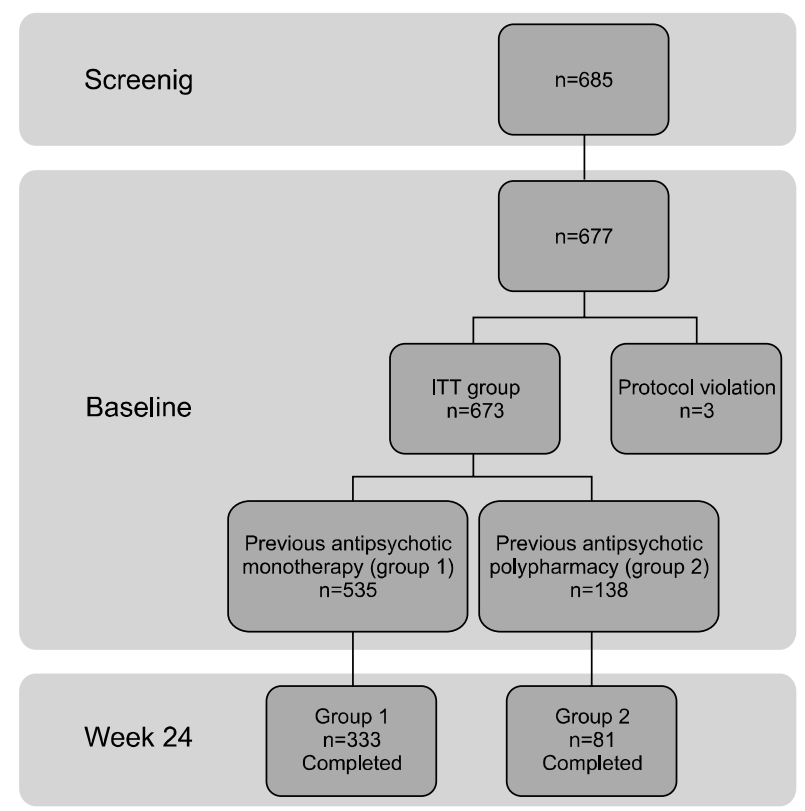

Fig. 1. Study flow schematic. ITT, intention to treat. 
(PSP) to study social functions. ${ }^{13)}$ This performs assessments by assessing four areas, namely, socially useful activities, personal and social relationships, self-care, disturbing and aggressive behavior. The PSP scores results from 1 to 100 points.

To measure EPS, we used the drug-induced extrapyramidal symptoms scale (DIEPSS), which was consists of eight individual assessment items and one global assessment. The eight items are; gait, bradykinesia, sialorrhea, muscle rigidity, tremor, akathisia, dystonia, and dyskinesia. To assess obesity, body mass index (BMI; defined as weight divided by height squared $\left[\mathrm{kg} / \mathrm{m}^{2}\right]$ ) were used.

\section{Statistical Analysis}

The independent $t$-test and the chi-square test were used to compare demographic and clinical variables in the monotherapy and polypharmacy groups at baseline (before switching to paliperidone ER). Kaplan-Meier analysis was used to compare group times to discontinuation of paliperidone ER. Cox proportional-hazards regression analysis was used to identify baseline risk factors that influence time to discontinuation of paliperidone ER, and repeated measure analysis of variance (ANOVA) was used to compare severity of psychopathology, EPS, and adverse events. To manage missing values created by drop-out or withdrawal, the last observation carried forward method (LOCF) was used (this method substitutes missing values with previous values). The statistical analysis was conducted using IBM SPSS Statistics ver. 19.0 (IBM Co., Armonk, NY, USA).

\section{RESULTS}

\section{Subjects}

A total of 673 patients were included in this pooledanalysis. At baseline, 535 (79.5\%) of the 673 had taken one antipsychotic medication (the monotherapy group), and 138 patients $(20.5 \%)$ were on polypharmacy (the polypharmacy group) (Table 1). In the monotherapy group, risperidone accounted for the largest number of patients $(n=248,46.4 \%)$, and in polypharmacy group, the numbers of patients that had previously taken typical antipsychotics plus atypical antipsychotics, atypical antipsychotics only, or typical antipsychotics only were 72 $(52.2 \%), 55(39.9 \%)$, and $11(8.0 \%)$, respectively. Regarding individual antipsychotics, the patients taking risperidone and haloperidol is the most common $(n=13,9.4 \%)$. Detailed information for individual antipsychotics previously prescribed is presented in Table 2 . Mean age and gender were similar in the two groups, but the polypharmacy group was more likely to experience severe hallucinations, more likely to be unemployed, and took higher doses of antipsychotics. No intergroup differences in other psychopathologies, EPS, or BMI were observed.

\section{Time to Discontinuation}

No difference in time to discontinuation of paliperidone ER was observed between the two groups (Fig. 2). Mean times to discontinuation of antipsychotics were 140.96 and 143.55 days in the days in the monotherapy and polypharmacy groups, respectively $(p=0.480)$. Cox propor-

Table 1. Baseline socio-demographic characteristics in the monotherapy and polypharmacy groups

\begin{tabular}{|c|c|c|c|c|}
\hline Characteristic & Monotherapy $(n=535)$ & Polypharmacy $(n=138)$ & $t\left(x^{2}\right)$ & $p$ value \\
\hline Sex (male) & $234(43.7)$ & $74(53.6)$ & 0.540 & 0.463 \\
\hline Age (year) & $36.23 \pm 10.509$ & $37.92 \pm 10.735$ & -1.740 & 0.082 \\
\hline Age of onset (year) & $26.74 \pm 9.058$ & $25.95 \pm 9.455$ & 0.866 & 0.387 \\
\hline Occupation (yes) & $99(19.0)$ & $19(12.4)$ & 6.672 & $0.036^{\dagger}$ \\
\hline Schizophrenia type & & & 1.462 & 0.833 \\
\hline Paranoid & $355(66.3)$ & $93(67.4)$ & & \\
\hline Disorganized & $10(1.9)$ & $2(1.4)$ & & \\
\hline Catatonic & $1(0.2)$ & $1(0.7)$ & & \\
\hline Undifferentiated & $135(25.2)$ & $34(24.6)$ & & \\
\hline Residual & $34(6.4)$ & $8(5.8)$ & & \\
\hline Krawiecka scale & $10.66 \pm 5.805$ & $11.50 \pm 6.343$ & -1.542 & 0.124 \\
\hline Depressed & $1.29 \pm 1.044$ & $1.19 \pm 1.068$ & 1.030 & 0.303 \\
\hline Hallucination & $1.36 \pm 1.421$ & $1.90 \pm 1.527$ & -4.015 & $<0.001^{\dagger}$ \\
\hline PSP & $57.12 \pm 15.591$ & $54.45 \pm 15.272$ & 1.871 & 0.062 \\
\hline Body mass index & $24.80 \pm 4.010$ & $25.22 \pm 4.140$ & -1.105 & 0.269 \\
\hline DIEPSS & $2.72 \pm 3.839$ & $3.31 \pm 4.020$ & -1.658 & 0.098 \\
\hline Dose of antipsychotics* (mg) & $301.01 \pm 239.630$ & $708.14 \pm 443.870$ & -10.392 & $<0.001^{\dagger}$ \\
\hline
\end{tabular}

Values are presented as number (\%) or mean \pm standard deviation.

${ }^{*}$ Chlorpromazine (100 mg) equivalent dose ${ }^{24)},{ }^{\dagger} p<0.05$.

PSP, personal and social performance scale; DIEPSS, drug-induced extrapyramidal symptoms scale. 
tional-hazard regression analysis showed that only PSP score influenced time to discontinuation (Table 3). Drop-out rates in the monotherapy and polypharmacy groups were $37.8 \%$ and $41.3 \%$, respectively 24 weeks $(p=0.299)$.

Table 2. Types and percentages of antipsychotics taken by the monotherapy and polypharmacy groups at baseline

\begin{tabular}{clc}
\hline Group & \multicolumn{1}{c}{ Types of antipsychotics } & Data \\
\hline Group 1 & Risperidone & $248(46.4)$ \\
& Olanzapine & $69(12.9)$ \\
& Aripiprazole & $62(11.6)$ \\
& Amisulpride & $41(7.7)$ \\
& Haloperidol & $34(6.4)$ \\
& Ziprasidone & $31(5.8)$ \\
Group 2 & Others & $50(9.2)$ \\
& Risperidone+haloperidol & $13(9.4)$ \\
& Risperidone+quetiapine & $10(7.2)$ \\
& Risperidone+chlorpromazine & $10(7.2)$ \\
& Amisulpride+haloperidol & $7(5.1)$ \\
& Risperidone+olanzapine & $6(4.3)$ \\
& Olanzapine+haloperidol & $6(4.3)$ \\
& Others & $86(62.5)$ \\
\hline
\end{tabular}

Values are presented as number (\%).

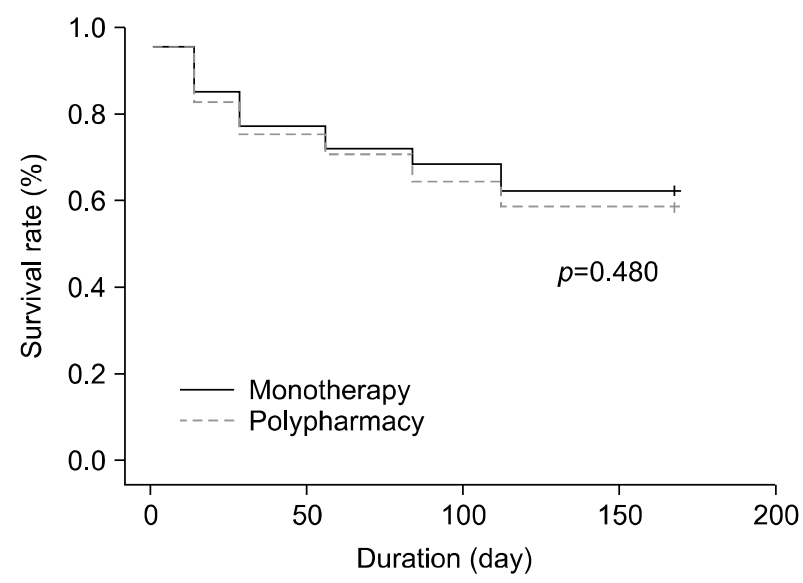

Fig. 2. Kaplan-Meier plot for time to discontinuation.

\section{Changes in Psychopathology and Adverse Events}

After 24 weeks from baseline, KS scores were significantly lower in both groups than at baseline. However, changes in KS scores in the two groups over this period were not significant different (Fig. 3, Table 4). Similarly, total DIEPSS scores reduced significantly in both groups, but no significant intergroup differences were found changes (Fig. 4, Table 4), and BMI significantly increased in both groups, but again without a significant intergroup difference (Fig. 5, Table 4).

Table 4. Psychopathologies, EPS, and body weight indices in the monotherapy and polypharmacy groups after 24 weeks

\begin{tabular}{lcc}
\hline \multicolumn{1}{c}{ Variable } & $F$ & P value \\
\hline Krawiecka scale & 26.067 & $<0.001^{*}$ \\
Krawiecka scale group & 1.389 & 0.217 \\
DIEPSS & 22.243 & $<0.001^{*}$ \\
DIEPSS group & 1.525 & 0.167 \\
Body mass index & 8.234 & $<0.001^{*}$ \\
Body mass index group & 0.314 & 0.930 \\
\hline
\end{tabular}

${ }^{*} p<0.05$; degree of freedom $=6$.

DIEPSS, drug-induced extrapyramidal symptoms scale.

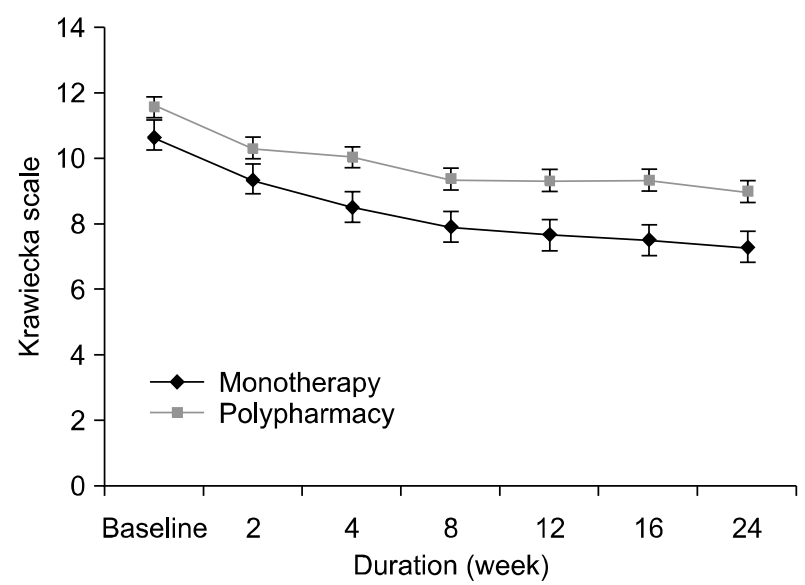

Fig. 3. Changes in total Krawiecka scale scores during the first 24 weeks.

Table 3. Factors influencing time to discontinuation of paliperidone extended-release therapy

\begin{tabular}{lcccccc}
\hline \multicolumn{1}{c}{ Variable } & $\mathrm{B}$ & $\mathrm{SE}$ & Wald & p-value & OR \\
\hline Group & -0.057 & 0.155 & 0.136 & 0.713 & 0.944 \\
Sex & -0.008 & 0.128 & 0.004 & 0.947 & 0.992 & $0.697-1.280$ \\
Age & -0.010 & 0.006 & 2.943 & 0.086 & $0.772-1.274$ \\
Occupation & 0.132 & 0.168 & 0.613 & 0.434 & $0.978-1.001$ \\
Depression (KS) & -0.023 & 0.063 & 0.138 & 0.710 & 0.977 \\
Hallucination (KS) & 0.022 & 0.047 & 0.210 & 0.647 & $0.820-1.587$ \\
PSP & -0.011 & 0.004 & 6.113 & $0.013^{*}$ & $0.964-1.105$ \\
\hline
\end{tabular}

${ }^{*} p<0.05$.

SE, standard error; OR, odds ratio; Cl, confidence interval; KS, Krawiecka scale; PSP, personal and social performance scale. 


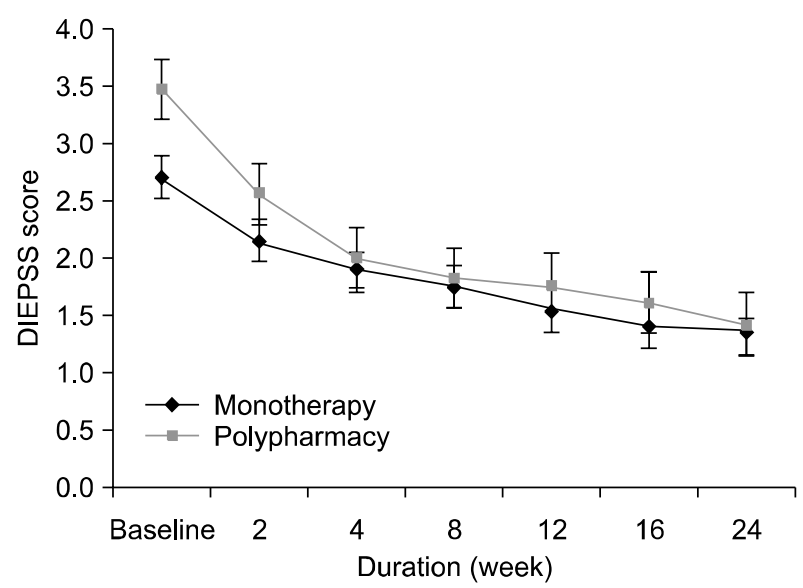

Fig. 4. Changes in total drug-induced extrapyramidal symptoms scale (DIEPSS) scores during the first 24 weeks.

\section{DISCUSSION}

In this study, we sought to determine whether the clinical courses of patients previously on antipsychotic monotherapy and polypharmacy differ. It was found that previous therapies did not influence time to discontinuation of paliperidone ER, psychopathology severity, or adverse events, including EPS and BMI. To the best of our knowledge, this is the first study to address possible differences between clinical courses and adverse events between patients previously on monotherapy or polypharmacy.

In terms of the comparative effectivenesses of antipsychotic monotherapy and polypharmacy, our results are in accord with two previous studies, which reported that the clinical effectiveness of antipsychotic monotherapy is comparable to that of polypharmacy. ${ }^{9,10)}$ Suzuki et al. ${ }^{9)}$ reported that switching to antipsychotic monotherapy was effective in 10 patients of $44(22.7 \%)$, but that the average global assessment functioning (GAF) scale of 44 patients after 24 weeks was $33.5 \%$, which was not significantly different from baseline. Furthermore, severity of illness (SOI) score of the clinical global impression (CGI) score decreased from 4.77 at baseline to 4.75 at 24 weeks after completion of switching, which was also not significant. ${ }^{9)}$ In a study conducted by Essock et al., ${ }^{10)}$ patients switched from polypharmacy to monotherapy and patients kept on polypharmacy had non-significantly different positive and negative syndrome scale (PANSS) score over 24 weeks as well as PANSS score in groups by time interaction. However, unlike previous studies, in the present study, we focused on the influences of number of previous antipsychotics before switching to paliperidone ER. In addition, to find the influence of the new medication, pre-

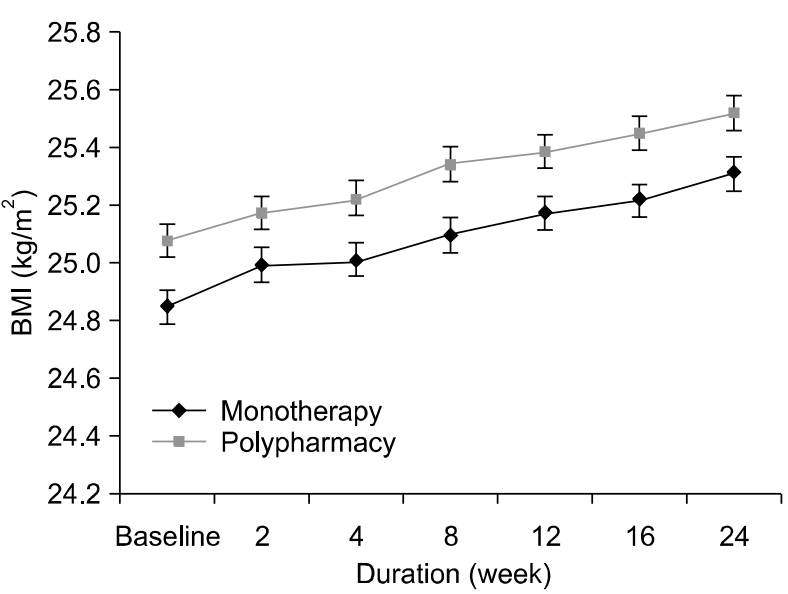

Fig. 5. Changes in body mass index (BMI) over the first 24 weeks.

vious study divided into two group; switching to new medication group and staying on previous medication group. ${ }^{14)}$ Unlike previous study, we switched to paliperidone ER in both polypharmacy group and monotherapy group.

As mentioned above, when clinicians find it necessary to modify an antipsychotic prescription, they usually switch to different type of antipsychotics, and in patients on two or more antipsychotics, clinicians tend to be concerned whether a newly prescribed single antipsychotics could be more effective than previous polypharmacy. ${ }^{8)}$ However, our results show that switching to paliperidone ER monotherapy is as effective in patients on previous polypharmacy. More specifically, effectiveness in terms of clinical symptoms and adverse events were not significantly different between patients on previous polypharmacy or monotherapy.

We also found that PSP at the baseline affected the discontinuation of antipsychotics, that is, good social function at baseline indicated a lower risk of discontinuation.

Furthermore, patients are usually expected to experience fewer side-effects, such as, EPS and weight increase when they switch from antipsychotic polypharmacy to monotherapy. Antipsychotic polypharmacy has been associated with higher mortality, drug interactions with various non-psychotropic agents, and higher cost. ${ }^{15-18)}$ However, in the present study, the polypharmacy group did not exhibit a significant decrease in DIEPSS or BMI as compared with the monotherapy group. Our results may represent that 24 weeks may not detect possible changes, and longer observation is needed.

This study also shows the superiority of paliperidone ER over previous antipsychotics in terms of efficacy and 
adverse effect. Paliperidone ER is an active metabolite of risperidone, and offers the benefits and effectiveness of risperidone, but is less metabolized in liver and exhibits fewer drug interactions. In addition, paliperidone ER maintains a stable concentration in blood for 24 hours. The merits of paliperidone ER in terms of its effectiveness, lack of side effects, and the prevention of recurrence have previously been described. ${ }^{19-22)}$

This study has several limitations. First, the original studies were not designed to compare the influences of previous antipsychotic medications, and thus, the dosages and type of previous antipsychotics were not controlled. Second, this study used the LOCF method that substituted missing values created by drop-outs with final values in the KS, the DIEPSS, and for BMI, and the relatively high drop-out rates encountered could have introduced study bias. Third, the duration of this study ( 24 weeks) may have prevented our detecting possible changes. We suggest a longer-term study should be conducted.

Nevertheless, the present study shows that switching to paliperidone ER had similar effects in antipsychotic polypharmacy and monotherapy patients in terms of treatment efficacy and side effects. Further studies are required to explore whether our results are applicable to antipsychotic types other than paliperidone ER.

\section{REFERENCES}

1. American Psychiatric Association; American Psychiatric Association. Task Force on DSM-IV. Diagnostic and statistical manual of mental disorders, fourth edition, text revision: DSM-IV. Washington, DC: American Psychiatric Association; 2000.

2. Buchanan RW, Kreyenbuhl J, Kelly DL, Noel JM, Boggs DL, Fischer BA, et al; Schizophrenia Patient Outcomes Research Team (PORT). The 2009 schizophrenia PORT psychopharmacological treatment recommendations and summary statements. Schizophr Bull 2010;36:71-93.

3. Treatment of schizophrenia 1999. The expert consensus guideline series. J Clin Psychiatry 1999;60(Suppl 11):3-80.

4. Lehman AF, Lieberman JA, Dixon LB, McGlashan TH, Miller AL, Perkins DO, et al; American Psychiatric Association; Steering Committee on Practice Guidelines. Practice guideline for the treatment of patients with schizophrenia, second edition. Am J Psychiatry 2004;161(2 Suppl):1-56.

5. Miller A, Hall CS, Buchanan RW, Buckley PF, Chiles JA, Conley RR, et al. The texas medication algorithm project antipsychotic algorithm for schizophrenia: 2003 update. J Clin Psychiatry 2004;65:500-508.

6. Essock SM, Covell NH, Davis SM, Stroup TS, Rosenheck RA, Lieberman JA. Effectiveness of switching antipsychotic medications. Am J Psychiatry 2006;163:2090-2095.

7. Tapp A, Wood AE, Secrest L, Erdmann J, Cubberley L, Kilzieh N. Combination antipsychotic therapy in clinical practice. Psychiatr Serv 2003;54:55-59.
8. Sernyak MJ, Rosenheck R. Clinicians' reasons for antipsychotic coprescribing. J Clin Psychiatry 2004;65:15971600.

9. Suzuki T, Uchida H, Tanaka KF, Nomura K, Takano H, Tanabe A, et al. Revising polypharmacy to a single antipsychotic regimen for patients with chronic schizophrenia. Int J Neuropsychopharmacol 2004;7:133-142.

10. Essock SM, Schooler NR, Stroup TS, McEvoy JP, Rojas I, Jackson C, et al; Schizophrenia Trials Network. Effectiveness of switching from antipsychotic polypharmacy to monotherapy. Am J Psychiatry 2011;168:702-708.

11. Tsutsumi C, Uchida H, Suzuki T, Watanabe K, Takeuchi H, Nakajima S, et al. The evolution of antipsychotic switch and polypharmacy in natural practice-a longitudinal perspective. Schizophr Res 2011;130:40-46.

12. Krawiecka M, Goldberg D, Vaughan M. A standardized psychiatric assessment scale for rating chronic psychotic patients. Acta Psychiatr Scand 1977;55:299-308.

13. Morosini PL, Magliano L, Brambilla L, Ugolini S, Pioli R. Development, reliability and acceptability of a new version of the DSM-IV Social and Occupational Functioning Assessment Scale (SOFAS) to assess routine social functioning. Acta Psychiatr Scand 2000;101:323-329.

14. Stroup TS, McEvoy JP, Ring KD, Hamer RH, LaVange LM, Swartz MS, et al; Schizophrenia Trials Network. A randomized trial examining the effectiveness of switching from olanzapine, quetiapine, or risperidone to aripiprazole to reduce metabolic risk: comparison of antipsychotics for metabolic problems (CAMP). Am J Psychiatry 2011;168: 947-956.

15. Essock SM, Covell NH, Leckman-Westin E, Lieberman JA, Sederer LI, Kealey E, et al. Identifying clinically questionable psychotropic prescribing practices for medicaid recipients in new york state. Psychiatr Serv 2009;60:15951602.

16. Waddington JL, Youssef HA, Kinsella A. Mortality in schizophrenia. Antipsychotic polypharmacy and absence of adjunctive anticholinergics over the course of a 10-year prospective study. Br J Psychiatry 1998;173:325-329.

17. Correll CU, Frederickson AM, Kane JM, Manu P. Does antipsychotic polypharmacy increase the risk for metabolic syndrome? Schizophr Res 2007;89:91-100.

18. Joukamaa M, Heliövaara M, Knekt P, Aromaa A, Raitasalo $\mathrm{R}$, Lehtinen V. Schizophrenia, neuroleptic medication and mortality. Br J Psychiatry 2006;188:122-127.

19. Kane J, Canas F, Kramer M, Ford L, Gassmann-Mayer C, Lim $\mathrm{P}$, et al. Treatment of schizophrenia with paliperidone extended-release tablets: a 6-week placebo-controlled trial. Schizophr Res 2007;90:147-161.

20. Emsley R, Berwaerts J, Eerdekens M, Kramer M, Lane R, Lim $\mathrm{P}$, et al. Efficacy and safety of oral paliperidone extended-release tablets in the treatment of acute schizophrenia: pooled data from three 52-week open-label studies. Int Clin Psychopharmacol 2008;23:343-356.

21. Canuso CM, Youssef EA, Bossie CA, Turkoz I, Schreiner A, Simpson GM. Paliperidone extended-release tablets in schizophrenia patients previously treated with risperidone. Int Clin Psychopharmacol 2008;23:209-215.

22. Marder SR, Kramer M, Ford L, Eerdekens E, Lim P, Eerdekens $\mathrm{M}$, et al. Efficacy and safety of paliperidone extended-release tablets: results of a 6-week, randomized, placebo-controlled study. Biol Psychiatry 2007;62:1363-1370. 